\title{
CHARACTERIZATIONS OF ERROR BOUNDS FOR LOWER SEMICONTINUOUS FUNCTIONS ON METRIC SPACES
}

\author{
Dominique AzÉ ${ }^{1}$ And JeAn-Nö̈l CORVElleC ${ }^{2}$
}

\begin{abstract}
Refining the variational method introduced in Azé et al. [Nonlinear Anal. 49 (2002) 643-670], we give characterizations of the existence of so-called global and local error bounds, for lower semicontinuous functions defined on complete metric spaces. We thus provide a systematic and synthetic approach to the subject, emphasizing the special case of convex functions defined on arbitrary Banach spaces (refining the abstract part of Azé and Corvellec [SIAM J. Optim. 12 (2002) 913-927], and the characterization of the local metric regularity of closed-graph multifunctions between complete metric spaces.
\end{abstract}

Mathematics Subject Classification. 49J52, 90C26, 90C25, $49 \mathrm{~J} 53$.

Received May 8, 2003.

\section{INTRODUCTION}

Let $X$ be a complete metric space, $f: X \rightarrow \mathbb{R} \cup\{+\infty\}$ be a lower semicontinuous function, and $\alpha \in \mathbb{R}$, $\beta \in \mathbb{R} \cup\{+\infty\}$ with $\alpha<\beta$. We say that $f$ has a global error bound between the levels $\alpha$ and $\beta$ if there exists a positive real number $\sigma$ such that

$$
f(x)-\alpha \geq \sigma \operatorname{dist}(x,[f \leq \alpha]) \quad \text { whenever } f(x) \in] \alpha, \beta[,
$$

where $[f \leq \alpha]:=\{x \in X: f(x) \leq \alpha\}$. In [5], and in the case when $\beta=+\infty$, we gave a sufficient condition for the existence of such global error bounds, in terms of an abstract notion of variational pair, the main property of which being an immediate consequence of Ekeland's variational principle [10]. Indeed, it is known since the pioneering work of Ioffe [13] in a more specific setting, that the variational principle is the natural tool for such questions. Two main examples of variational pairs were given in [5], the first one involving the strong slope of De Giorgi, Marino, and Tosques [9], the second one being related to subdifferentials of lower semicontinuous functions defined on Banach spaces.

However, it turns out that the strong slope indeed yields a characterization of global error bounds on the one hand (as was observed in [5]), and that it can be appropriately compared with subdifferential operators on the other hand, as explicated in Section 4 of this paper. This suggests that the strong slope is indeed the adequate notion in order to deal with error bounds (either global or local), yielding both accurate and general results.

\footnotetext{
Keywords and phrases. Error bounds, strong slope, variational principle, metric regularity.

1 UMR CNRS MIP, Université Paul Sabatier, 118 route de Narbonne, 31062 Toulouse Cedex, France;

e-mail: aze@mip.ups-tlse.fr

2 Laboratoire MANO, Université de Perpignan, 52 avenue de Villeneuve, 66860 Perpignan Cedex, France.
} 
This fact was also stressed by Ioffe in [16] after he became aware of the paper [5], in the related context of local metric regularity.

Taking these considerations into account, we provide in this paper a refined approach, with respect to [5], to the characterization of the existence of error bounds, in the afore-mentioned setting. These refinements concern the statements, the presentation, and the proofs as well. The basic notions, tools, and abstract principle (see Th. 2.1) are given in Section 2.

In Section 3, we specialize our main abstract result to the case of convex functions, which has been the object of numerous studies, starting with the pioneering work of Hoffman [12]. In this case, a constant $\sigma$ such that (1) holds true is accordingly called a Hoffman constant, and we refine and extend the abstract part of our previous paper [4]. This allows to recover, and often sharpen, various earlier results on characterization, or on sufficient conditions for the existence, of a global error bound. Moreover, our systematic approach naturally provides simple proofs and, in our opinion, sheds a new light on the matter.

In Section 5, we give local versions of our main abstract result, thus dealing with what may be called local error bounds. As illustrations of these results, we give characterizations of so-called weak sharp local minima, and of the local metric regularity for closed-graph multifunctions between complete metric spaces, that we compare with the corresponding result of [16]. Here again, our results refine and somewhat clarify those already given in [5]. As a whole, we hope that this paper can provide a useful, synthetic view of the subject matter.

Throughout the paper, we let $X$ be a metric space endowed with the metric $d$, and $f: X \rightarrow \mathbb{R} \cup\{+\infty\}$ be a lower semicontinuous function. For $U \subset X$ and $r \in] 0,+\infty]$ (resp., $r \in\left[0,+\infty\left[\right.\right.$ ), we denote by $B_{r}(U)$ (resp., $\left.\bar{B}_{r}(U)\right)$ the open (resp., closed) r-neighborhood of $U$ :

$$
B_{r}(U):=\{x \in X: d(x, U)<r\}, \quad \bar{B}_{r}(U):=\{x \in X: d(x, U) \leq r\},
$$

where

$$
d(x, U):=\inf \{d(x, y): y \in U\}
$$

with the convention that $d(x, \emptyset)=+\infty$ (according to the general convention inf $\emptyset=+\infty)$. If $U=\{x\}$, we simply write $B_{r}(x), \bar{B}_{r}(x)$. For $\alpha \in \mathbb{R}$ and $\beta \in \mathbb{R} \cup\{+\infty\}$, we let:

$$
[f \leq \alpha]:=\{x \in X: f(x) \leq \alpha\}, \quad[f<\beta]:=\{x \in X: f(x)<\beta\}
$$

denote respectively the closed and open sublevel sets of $f$, and if $\alpha<\beta$, we further let

$$
[\alpha<f<\beta]:=[f<\beta] \backslash[f \leq \alpha]
$$

denote the "slice" between $\alpha$ and $\beta$. If $\beta=+\infty$, we shall rather write:

$$
[f>\alpha]:=[\alpha<f<+\infty], \quad \operatorname{dom} f:=[f<+\infty]
$$

and say, as usual, that $f$ is proper if $\operatorname{dom} f \neq \emptyset$.

\section{Characterization of GLOBAl ERror BOUnds}

We first recall the notion of strong slope introduced by De Giorgi, Marino, and Tosques in [9].

Definition 2.1. Let $f: X \rightarrow \mathbb{R} \cup\{+\infty\}$ be a lower semicontinuous function, and let $x \in \operatorname{dom} f$. Set:

$$
|\nabla f|(x):= \begin{cases}0 & \text { if } x \text { is a local minimum of } f, \\ \limsup _{y \rightarrow x} \frac{f(x)-f(y)}{d(x, y)} & \text { otherwise. }\end{cases}
$$

For $x \notin \operatorname{dom} f$, let $|\nabla f|(x):=+\infty$. The nonnegative extended real number $|\nabla f|(x)$ is called the strong slope of $f$ at $x$. 
The following notion plays a central role in the paper.

Definition 2.2. Let $f: X \rightarrow \mathbb{R} \cup\{+\infty\}$ be a lower semicontinuous function. For $\alpha \in \mathbb{R}, \beta \in \mathbb{R} \cup\{+\infty\}$ with $\alpha<\beta$, we let $\sigma_{\alpha, \beta}(f)$ denote the supremum of the $\sigma$ 's in $[0,+\infty[$ such that

$$
f(x)-\alpha \geq \sigma d(x,[f \leq \alpha]) \text { for every } x \in[\alpha<f<\beta]
$$

with the conventions:

$$
\sigma_{\alpha, \beta}(f)=\left\{\begin{array}{lr}
0 & \text { if }[f \leq \alpha]=\emptyset \text { and }[\alpha<f<\beta] \neq \emptyset \\
+\infty & \text { if }[\alpha<f<\beta]=\emptyset
\end{array}\right.
$$

Of course, we have:

$$
\sigma_{\alpha, \beta}(f)=\inf _{x \in[\alpha<f<\beta]} \frac{f(x)-\alpha}{d(x,[f \leq \alpha])}
$$

(taking the above conventions into account). If $\beta=+\infty$, we simply write:

$$
\sigma_{\alpha}(f):=\sigma_{\alpha,+\infty}(f)
$$

Finally, we say that $f$ has a global error bound between the levels $\alpha$ and $\beta$, if $\sigma_{\alpha, \beta}(f)>0$.

We first note the following immediate consequence of the above two definitions, which gives, in particular, a necessary condition for the existence of a global error bound.

Proposition 2.1. Let $f: X \rightarrow \mathbb{R} \cup\{+\infty\}$ be a lower semicontinuous function, $U$ be a subset of $X$, and $\alpha \in \mathbb{R}$, $\beta \in \mathbb{R} \cup\{+\infty\}$ with $\alpha<\beta$. Then:

$$
\inf _{U \cap[\alpha<f<\beta]}|\nabla f| \geq \inf _{\alpha \leq \gamma<\beta} \inf _{\alpha \in U \cap[\gamma<f<\beta]} \frac{f(x)-\gamma}{d(x,[f \leq \gamma])} .
$$

In particular:

$$
\inf _{[\alpha<f<\beta]}|\nabla f| \geq \inf _{\alpha \leq \gamma<\beta} \sigma_{\gamma, \beta}(f) .
$$

Proof. We may assume that the left-hand side of the inequality is finite, so that $U \cap[\alpha<f<\beta] \neq \emptyset$, and that the right-hand side is positive, so that $[f \leq \alpha] \neq \emptyset$ (we use similar conventions as in Def. 2.2). Let $\sigma>0$ be such that

$$
\inf _{x \in U \cap[\gamma<f<\beta]} \frac{f(x)-\gamma}{d(x,[f \leq \gamma])}>\sigma \quad \text { for every } \gamma \in[\alpha, \beta[
$$

let $x \in U \cap[\alpha<f<\beta]$, and set $\gamma_{n}:=f(x)-1 / n$ for $n \in \mathbb{N}$ large enough so that $\gamma_{n} \geq \alpha$. For each $n \in \mathbb{N}$, let $x_{n} \in\left[f \leq \gamma_{n}\right]$ be such that $f(x)-\gamma_{n} \geq \sigma d\left(x, x_{n}\right)$, according to (4). Then, we have:

$$
0<d\left(x, x_{n}\right) \leq \frac{f(x)-\gamma_{n}}{\sigma} \rightarrow 0 \quad \text { as } n \rightarrow \infty
$$

so that $x$ is not a local minimum of $f$, and

$$
\frac{f(x)-f\left(x_{n}\right)}{d\left(x, x_{n}\right)} \geq \frac{f(x)-\gamma_{n}}{d\left(x, x_{n}\right)} \geq \sigma
$$

showing that $|\nabla f|(x) \geq \sigma$, and the conclusion follows.

The main tool which is needed for our purposes is, of course, Ekeland's variational principle [10], of which we now recall an appropriate version, as well as an essential consequence in terms of the strong slope. 
Proposition 2.2. Let $X$ be complete, $f: X \rightarrow \mathbb{R} \cup\{+\infty\}$ be a (proper) lower semicontinuous function, and let $\bar{x} \in X, \sigma>0$, and $r>0$, be such that:

$$
f(\bar{x}) \leq \inf _{X} f+\sigma r .
$$

Then, there exists $x \in \bar{B}_{r}(\bar{x})$ such that $f(x) \leq f(\bar{x})$ and

$$
f(x)<f(y)+\sigma d(x, y) \quad \text { for every } y \in X \backslash\{x\} .
$$

Corollary 2.1. Let $X$ be complete, $f: X \rightarrow \mathbb{R} \cup\{+\infty\}$ be a lower semicontinuous function, and let $\bar{x} \in X$, $\sigma>0$, and $r>0$, be such that:

$$
f(\bar{x})<\inf _{\bar{B}_{r}(\bar{x})} f+\sigma r .
$$

Then, there exists $x \in B_{r}(\bar{x})$ such that $f(x) \leq f(\bar{x})$ and $|\nabla f|(x)<\sigma$.

Proof. Let $0<\sigma^{\prime}<\sigma$ and $0<r^{\prime}<r$ be such that

$$
f(\bar{x}) \leq \inf _{\bar{B}_{r}(\bar{x})} f+\sigma^{\prime} r^{\prime} .
$$

Applying Proposition 2.2 with $X:=\bar{B}_{r}(\bar{x})$, we find $x \in \bar{B}_{r^{\prime}}(\bar{x}) \subset B_{r}(\bar{x})$ with $f(x) \leq f(\bar{x})$ and $\left|\nabla f_{\mid \bar{B}_{r}(\bar{x})}\right|(x)=$ $|\nabla f|(x) \leq \sigma^{\prime}<\sigma$, as follows from (5) and the definition of the strong slope.

Remark 2.1. (a) We want to emphasize here that, by definition, the strong slope gives the best quantitative estimate of the (lower) behavior of $f$ at the "approximate stationary point" $x$ given by the variational principle. We shall further comment on this fact below, after stating our main abstract result.

(b) Since $f$ is lower semicontinuous, we readily see, from the above corollary, that for any $-\infty<\alpha<\beta \leq$ $+\infty$, we have that $[\alpha<f<\beta] \cap \operatorname{dom}|\nabla f|$ is dense in $[\alpha<f<\beta]$, and that, if the latter set is nonempty, while $[f \leq \alpha]=\emptyset$ (so that $\inf _{X} f \geq \alpha$ ), then $\inf _{[\alpha<f<\beta]}|\nabla f|=0$. Thus:

$$
0<\inf _{[\alpha<f<\beta]}|\nabla f|<+\infty \Longrightarrow[f \leq \alpha] \neq \emptyset .
$$

Indeed, this is contained in the following proposition (that we shall use in Sect. 5), letting $U:=X, \sigma:=$ $\inf _{[\alpha<f<\beta]}|\nabla f|$, and $\rho:=\frac{\beta-\alpha}{\sigma}(=+\infty$ if $\beta=+\infty)$ in this result.

Proposition 2.3. Let $X$ be complete, $f: X \rightarrow \mathbb{R} \cup\{+\infty\}$ be a lower semicontinuous function, $U$ be a subset of $X, \alpha \in \mathbb{R}$, and $\sigma, \rho>0$. Assume that $U \cap[f<\alpha+\sigma \rho] \neq \emptyset$ and that:

$$
\inf _{B_{\rho}(U) \cap[\alpha<f<\alpha+\sigma \rho]}|\nabla f| \geq \sigma .
$$

Then, $[f \leq \alpha] \neq \emptyset$.

Proof. Assume, for a contradiction, that $[f \leq \alpha]=\emptyset$, and let $\bar{x} \in U \cap[\alpha<f<\alpha+\sigma \rho]$. We have:

$$
f(\bar{x})<\alpha+\sigma \rho \leq \inf _{X} f+\sigma \rho,
$$

and, according to Corollary 2.1, we find $x \in B_{\rho}(\bar{x}) \subset B_{\rho}(U)$ with $\alpha<f(x) \leq f(\bar{x})<\alpha+\sigma \rho$ and $|\nabla f|(x)<\sigma$, contradicting our assumption.

We now state our main result. 
Theorem 2.1. Let $X$ be complete, $f: X \rightarrow \mathbb{R} \cup\{+\infty\}$ be a lower semicontinuous function, and $\alpha \in \mathbb{R}$, $\beta \in \mathbb{R} \cup\{+\infty\}$ with $\alpha<\beta$. Then,

$$
\inf _{[\alpha<f<\beta]}|\nabla f|=\inf _{\alpha \leq \gamma<\beta} \sigma_{\gamma, \beta}(f)=\inf _{\alpha \leq \gamma<\beta} \inf _{x \in[\gamma<f<\beta]} \frac{f(x)-\gamma}{d(x,[f \leq \gamma])} .
$$

Proof. According to (2) and (3), we just have to show that

$$
\sigma_{\gamma, \beta}(f) \geq \inf _{[\alpha<f<\beta]}|\nabla f| \quad \text { for any } \alpha \leq \gamma<\beta
$$

Let $\gamma \in\left[\alpha, \beta\right.$ [ be fixed: we may of course assume that $\sigma_{\gamma, \beta}(f)<+\infty$, so that $[\gamma<f<\beta] \neq \emptyset$, and that $\inf _{[\alpha<f<\beta]}|\nabla f|>0$, so that $[f \leq \alpha] \neq \emptyset$ (see Rem. 2.1(b)). Consider a real number $\sigma>\sigma_{\gamma, \beta}(f)$ and $\bar{x} \in[\gamma<f<\beta]$ with

$$
f(\bar{x})-\gamma<\sigma d(\bar{x},[f \leq \gamma]) .
$$

Set $r:=d(\bar{x},[f \leq \gamma])>0, g:=(f-\gamma)^{+} \geq 0$, i.e., $g(x):=\max \{f(x)-\gamma, 0\}$, so that

$$
g(\bar{x})<\inf _{\bar{B}_{r}(\bar{x})} g+\sigma r
$$

According to Corollary 2.1, we find $x \in B_{r}(\bar{x})$ with $g(x) \leq g(\bar{x})$ and $|\nabla g|(x)<\sigma$. By definition of $r$, we see that $f(x)>\gamma$, so that $f(x)=g(x)+\gamma \leq g(\bar{x})+\gamma=f(\bar{x})<\beta$ and $|\nabla f|(x)=|\nabla g|(x)<\sigma$. Thus, $\inf _{[\alpha<f<\beta]}|\nabla f|<\sigma$, and the conclusion follows.

Remark 2.2. (a) In the case when $\beta=+\infty$, Theorem 2.1 was given in [4], (Th. 2.4) (and can be traced back to [5], (Prop. 2.4, Th. 3.1, Rem. 3.2)). The generalization to arbitrary "slices" $[\alpha<f<\beta]$ is for free: it is obtained using also the fact (which is not needed if $\beta=+\infty$ ) that $f(x) \leq f(\bar{x})$ in Corollary 2.1, so that indeed, all the informations given by the variational principle are used. This generalization is useful, too, as we shall see in the next sections.

Let us already mention that Theorem 2.1 shows that

$$
+\infty>\inf _{[\alpha<f<\beta]}|\nabla f| \geq \sigma>0 \Longrightarrow[f \leq \alpha] \neq \emptyset \quad \text { and } \quad \sigma_{\gamma, \beta}(f) \geq \sigma \quad \forall \gamma \in[\alpha, \beta[,
$$

that is, it gives a sufficient condition for the existence of a global error bound for $f$ between the levels $\gamma$ and $\beta$, for any $\alpha \leq \gamma<\beta$. It also shows (indeed) that the best possible estimates for global error bounds are given in terms of the strong slope, yielding a necessary condition, and suggesting that other sufficient conditions for a global error bound involving notions such as subdifferentials or directional derivatives, should be a consequence of (6) (recall Rem. 2.1(a)): this will be detailed in Section 4, after we treat the case of convex functions $f$ in Section 3.

(b) Recently, Wu and Ye established in [26] (Th. 3) the following sufficient condition for a global error bound, for a (proper) lower semicontinuous function $f: X \rightarrow \mathbb{R} \cup\{+\infty\}$ defined on the complete metric space $X$ (we use our notations):

let $0<\varepsilon \leq+\infty$ and $\sigma>0$, assume that $[f<\varepsilon] \neq \emptyset$, and that for each $x \in[0<f<\varepsilon]$ there exists $y \in X$ with $f(y) \geq 0$ and

$$
f(x)-f(y) \geq \sigma d(x, y)>0 .
$$

Then, $[f \leq 0] \neq \emptyset$ and $\sigma_{0, \varepsilon}(f) \geq \sigma$. 
While the proof given in [26] is rather involved, it is easy to see that this can be established in a straightforward way, arguing similarly as in Proposition 2.3 and in Theorem 2.1, but using (the stronger) Proposition 2.2 instead of Corollary 2.1. Indeed, if $\sigma_{0, \varepsilon}(f)<\sigma$, we find $\bar{x} \in[0<f<\varepsilon]$ and a positive real $r \leq d(\bar{x},[f \leq 0])$ such that

$$
g(\bar{x})<\inf _{X} g+\sigma r
$$

where $g:=f^{+}$. According to Proposition 2.2, we find $x \in B_{r}(\bar{x})$ such that $g(x) \leq g(\bar{x})$ and

$$
g(x)-g(y)<\sigma d(x, y) \quad \text { for every } y \neq x .
$$

Then, $0<f(x)=g(x)<\varepsilon$ and $f(x)-f(y)<\sigma d(x, y)$ for every $y \in X$ such that $f(y)=g(y) \geq 0$. Hence the result.

However, such result does not allow to obtain a characterization of global error bounds - but in the convex case: see Proposition 3.1.

\section{The CONVEX CASE}

In this section, $X$ is a Banach space, endowed with a norm $\|\cdot\|$. We denote by $X^{*}$ the topological dual of $X$, and by $d_{*}$ the metric associated with the dual norm. Recall that if $f: X \rightarrow \mathbb{R} \cup\{+\infty\}$ is a convex lower semicontinuous function, the (Fenchel) subdifferential of $f$ at $x \in \operatorname{dom} f$ is given by:

$$
\partial f(x)=\left\{x^{*} \in X^{*}: f(y)-f(x) \geq\left\langle x^{*}, y-x\right\rangle \forall y \in X\right\},
$$

while the directional derivative of $f$ at $x$ in the direction $y \in X$ is:

$$
f^{\prime}(x ; y):=\lim _{\lambda \searrow 0} \frac{f(x+\lambda y)-f(x)}{\lambda}=\inf _{\lambda>0} \frac{f(x+\lambda y)-f(x)}{\lambda} .
$$

If $x \notin \operatorname{dom} f$, we let (as usual) $\partial f(x)=\emptyset, f^{\prime}(x ; y)=-\infty$ for any $y \in X$.

Proposition 3.1. Let $X$ be a Banach space, $f: X \rightarrow \mathbb{R} \cup\{+\infty\}$ be a proper, convex, and lower semicontinuous function, and $x \in X$ which is not a minimum point of $f$. Then:

$$
|\nabla f|(x)=\sup _{f(z)<f(x)} \frac{f(x)-f(z)}{\|x-z\|}=\sup _{f(z)<f(x)} \frac{-f^{\prime}(x ; z-x)}{\|x-z\|}=d_{*}(0, \partial f(x)) .
$$

Proof. These equalities are well known: for example, the last two are mentioned in [28] (Prop. 3.2), the fact that $d_{*}(0, \partial f(x))=\sup _{f(z)<f(x)} \frac{f(x)-f(z)}{\|x-z\|}$ is in [24], while the fact that $|\nabla f|(x)=d_{*}(0, \partial f(x))$ (which is true for all $x \in X$, that is, also when $x$ is a local minimum point of $f$ ) is mentioned in [4] (Prop. 2.2). We give a quick proof for completeness and for the reader's convenience. Let $x \in \operatorname{dom} f$ which is not a minimum (hence, not a local minimum) point of $f$. For every $z \in X$ with $f(z)<f(x)$ and for every $\lambda \in] 0,1]$, we have:

$$
\frac{f(x)-f(z)}{\|x-z\|} \leq \frac{f(x)-f(x+\lambda(z-x))}{\lambda\|x-z\|} \leq\left\|x^{*}\right\|_{*}
$$

whenever $x^{*} \in \partial f(x)$, and thus:

$$
|\nabla f|(x) \leq \sup _{f(z)<f(x)} \frac{f(x)-f(z)}{\|x-z\|} \leq \sup _{f(z)<f(x)} \frac{-f^{\prime}(x ; z-x)}{\|x-z\|} \leq d_{*}(0, \partial f(x)),
$$


the last inequality being trivial if $\partial f(x)=\emptyset$. On the other hand, let $0<\sigma<d_{*}(0, \partial f(x))$. Then, $x$ is not a minimum point of the (convex) function $z \mapsto f(z)+\sigma\|x-z\|-$ for, otherwise, from standard convex calculus, we have $0 \in \partial f(x)+\sigma \bar{B}_{*}$, where $\bar{B}_{*}$ denotes the closed unit ball of $X^{*}$, contradicting the choice of $\sigma$. Let thus $z \in X$ be such that $f(z)+\sigma\|x-z\|<f(x)$, so that for every $\lambda \in] 0,1]$ :

$$
\frac{f(x)-f(x+\lambda(z-x))}{\lambda\|x-z\|}>\sigma
$$

showing that $|\nabla f|(x) \geq \sigma$, whence $|\nabla f|(x) \geq d_{*}(0, \partial f(x))$.

We observe in the following that for a convex function, the slope decreases with altitude. For $\alpha \in \mathbb{R}$, we let:

$$
[f=\alpha]:=\{x \in X: f(x)=\alpha\} .
$$

Proposition 3.2. Let $X$ be a Banach space, $f: X \rightarrow \mathbb{R} \cup\{+\infty\}$ be a convex, lower semicontinuous function, and $\alpha \in \mathbb{R}, \beta \in \mathbb{R} \cup\{+\infty\}$ with $\alpha<\beta$. If $[f \leq \alpha] \neq \emptyset$, then:

$$
\inf _{[f>\alpha]}|\nabla f| \geq \inf _{[f=\alpha]}|\nabla f| .
$$

Consequently, if $[f<\beta] \neq \emptyset$, then:

$$
\inf _{[f>\alpha]}|\nabla f|=\inf _{[\alpha<f<\beta]}|\nabla f| .
$$

Proof. We may assume that $\inf _{[f=\alpha]}|\nabla f|>0$, so that $\alpha>\inf _{X} f$, and that $\inf _{[f>\alpha]}|\nabla f|<+\infty$. Let thus $x \in[f>\alpha]$ and $\sigma>0$ with $\sigma>|\nabla f|(x)$. For $y \in[f \leq \alpha]$, set:

$$
g(y):=f(y)+\sigma\|y-x\|,
$$

so that $g>f(x)$ - for, otherwise, let $y \in[f \leq \alpha]$ be such that $f(y)+\sigma\|y-x\| \leq f(x)$, then

$$
|\nabla f|(x) \geq \frac{f(x)-f(y)}{\|x-y\|} \geq \sigma,
$$

a contradiction. Let now $\varepsilon \in] 0, \sigma-|\nabla f|(x)\left[\right.$. According to Proposition 2.2, there exists $y_{\varepsilon} \in[f \leq \alpha]$ such that $g(y) \geq g\left(y_{\varepsilon}\right)-\varepsilon\left\|y-y_{\varepsilon}\right\|$ for all $y \in[f \leq \alpha]$, that is:

$$
f(y)+\sigma\|y-x\| \geq f\left(y_{\varepsilon}\right)+\sigma\left\|y_{\varepsilon}-x\right\|-\varepsilon\left\|y-y_{\varepsilon}\right\| \text { for all } y \in[f \leq \alpha] .
$$

We claim that $f\left(y_{\varepsilon}\right)=\alpha$. For, if $f\left(y_{\varepsilon}\right)<\alpha$, let $z_{\varepsilon}$ be a point in the open segment $] x, y_{\varepsilon}\left[\right.$ with $f\left(z_{\varepsilon}\right)=\alpha$; then, writing (7) for $y:=z_{\varepsilon}$ yields:

whence

$$
f\left(z_{\varepsilon}\right)-f\left(y_{\varepsilon}\right) \geq(\sigma-\varepsilon)\left\|z_{\varepsilon}-y_{\varepsilon}\right\|,
$$

$$
|\nabla f|(x) \geq \frac{f(x)-f\left(z_{\varepsilon}\right)}{\left\|x-z_{\varepsilon}\right\|} \geq \frac{f\left(z_{\varepsilon}\right)-f\left(y_{\varepsilon}\right)}{\left\|z_{\varepsilon}-y_{\varepsilon}\right\|} \geq \sigma-\varepsilon
$$

a contradiction. Now, we derive from (7) again that for all $y \in[f \leq \alpha]$ :

$$
f\left(y_{\varepsilon}\right)-f(y) \leq \sigma\left(\|y-x\|-\left\|y_{\varepsilon}-x\right\|\right)+\varepsilon\left\|y-y_{\varepsilon}\right\| \leq(\sigma+\varepsilon)\left\|y-y_{\varepsilon}\right\|,
$$

so that

$$
|\nabla f|\left(y_{\varepsilon}\right)=\sup _{f(y)<f\left(y_{\varepsilon}\right)} \frac{f\left(y_{\varepsilon}\right)-f(y)}{\left\|y_{\varepsilon}-y\right\|} \leq \sigma+\varepsilon
$$

which shows that $\inf _{[f=\alpha]}|\nabla f| \leq \sigma$, since $\varepsilon$ is arbitrarily small, and the first assertion follows. 
In order to get the second assertion, we need to show that

$$
\inf _{[f>\alpha]}|\nabla f| \geq \inf _{[\alpha<f<\beta]}|\nabla f|
$$

whenever $\beta \in \mathbb{R}$. We may assume that $[f>\alpha] \cap \operatorname{dom} f \neq \emptyset$, so that $[\alpha<f<\beta] \neq \emptyset$, since $[f<\beta] \neq \emptyset$. Thus, let $\gamma \in] \alpha, \beta[$ with $[f=\gamma] \neq \emptyset$, using the first assertion we get:

$$
\inf _{[f>\gamma]}|\nabla f| \geq \inf _{[f=\gamma]}|\nabla f| \geq \inf _{[\alpha<f<\beta]}|\nabla f|,
$$

and the conclusion follows.

Remark 3.1. (a) In the case when for every $x \in[f>\alpha]$ there exists a projection on $[f \leq \alpha]$ (for example, if the Banach space $X$ is reflexive), the proof of the first assertion in Proposition 3.2 is straightforward: indeed, let $0<\sigma<\inf _{[f=\alpha]}|\nabla f|$ and $x \in[f>\alpha]$, and let $y \in[f=\alpha]$ be such that $\|x-y\|=d(x,[f \leq \alpha])$. Let then $z \in[f<\alpha]$ be such that

$$
\frac{f(y)-f(z)}{\|y-z\|}=\frac{\alpha-f(z)}{\|y-z\|} \geq \sigma
$$

and let $\left.y^{\prime} \in\right] x, z\left[\cap[f=\alpha]\right.$. Since $\|x-y\| \leq\left\|x-y^{\prime}\right\|$, we have $\left\|y^{\prime}-z\right\| \leq\|y-z\|$, so that

$$
\frac{f(x)-f(z)}{\|x-z\|} \geq \frac{f\left(y^{\prime}\right)-f(z)}{\left\|y^{\prime}-z\right\|}=\frac{\alpha-f(z)}{\|y-z\|} \frac{\|y-z\|}{\left\|y^{\prime}-z\right\|} \geq \sigma,
$$

hence $|\nabla f|(x) \geq \sigma$ and the conclusion follows.

(b) In the case when $X=\mathbb{R}^{n}$, and under the additional (but unnecessary) assumption that $[f>\alpha] \cap \operatorname{dom} f$ is contained in the relative interior of $\operatorname{dom} f$, an equivalent formulation of the first assertion in Proposition 3.2 can be found in [3] (Prop. 4.2.1).

Proposition 3.3. Let $X$ be a Banach space, $f: X \rightarrow \mathbb{R} \cup\{+\infty\}$ be a proper, convex, and lower semicontinuous function, and $\alpha \in \mathbb{R}, \beta \in \mathbb{R} \cup\{+\infty\}$ with $\alpha<\beta$. Then:

$$
\sigma_{\alpha, \beta}(f)=\inf _{\alpha \leq \gamma<\beta} \sigma_{\gamma, \beta}(f) .
$$

Proof. This is a slight variant of [4] (Prop. 2.2(b)), where $\beta=+\infty$, so we just sketch the proof. Given $\gamma \in] \alpha, \beta[$, we only need to show that $\sigma_{\gamma, \beta}(f) \geq \sigma_{\alpha, \beta}(f)$, assuming that $\sigma_{\gamma, \beta}(f)<+\infty$, so that $[\gamma<f<\beta] \neq \emptyset$, and that $\sigma_{\alpha, \beta}(f)>0$, so that $[f \leq \alpha] \neq \emptyset$. Let $0<\sigma<\sigma_{\alpha, \beta}(f), x \in[\gamma<f<\beta]$, and $\varepsilon>0$ be such that $\sigma(1+\varepsilon) \leq \sigma_{\alpha, \beta}(f)$. Let then $y \in[f \leq \alpha]$ be such that $\|x-y\| \leq(1+\varepsilon) d(x,[f \leq \alpha])$, so that $f(x)-\alpha \geq \sigma\|x-y\|$. Finally, thanks to the convexity of $f$, and considering a point $z \in] x, y[\cap[f=\gamma]$, we obtain:

$$
\begin{aligned}
f(x)-\gamma & \geq f(x)\left(1-\frac{\|z-y\|}{\|x-y\|}\right)-\frac{\|z-x\|}{\|x-y\|} \alpha \\
& =(f(x)-\alpha) \frac{\|x-z\|}{\|x-y\|} \geq \sigma\|x-z\| \geq \sigma d(x,[f \leq \gamma]),
\end{aligned}
$$

showing that $\sigma_{\gamma, \beta}(f) \geq \sigma$, and the conclusion. 
We now state the main result of this section.

Theorem 3.1. Let $X$ be a Banach space, $f: X \rightarrow \mathbb{R} \cup\{+\infty\}$ be a proper, convex, and lower semicontinuous function, and $\alpha \in \mathbb{R}, \beta \in \mathbb{R} \cup\{+\infty\}$ with $\alpha<\beta$. Then:

$$
\inf _{x \in[\alpha<f<\beta]} \frac{f(x)-\alpha}{d(x,[f \leq \alpha])}=\inf _{x \in[\alpha<f<\beta]} d_{*}(0, \partial f(x)) .
$$

Moreover, if $[f<\beta] \neq \emptyset$, then:

$$
\inf _{x \in[f>\alpha]} \frac{f(x)-\alpha}{d(x,[f \leq \alpha])}=\inf _{x \in[\alpha<f<\beta]} d_{*}(0, \partial f(x))
$$

$(=+\infty$ if $[\alpha<f<\beta]=\emptyset ;=0$ if $[f \leq \alpha]=\emptyset)$.

Proof. The first conclusion is obtained by combining Theorem 2.1, Proposition 3.1, and Proposition 3.3. The second one is obtained using the first one with $\beta:=+\infty$, the second part of Proposition 3.2, and Proposition 3.1 again.

Remark 3.2. (a) In the case when $\beta=+\infty$, the first conclusion of Theorem 3.1 was already given in [4] (Th. 2.6), while the second one was only mentioned in a particular case (see [4], (Prop. 3.5)), which was sufficient (and used) for the main purposes of [4], as described in the title of that paper. Since then, the conclusions of Theorem 3.1 have been obtained in [26] (Th. 7), where they are expressed in a more customary way, through the equivalence of several properties.

(b) To our knowledge, the first characterization of the existence of a global error bound in the convex case is [8] (Th. 5.1), where it is shown that if $\operatorname{argmin} f \neq \emptyset, \alpha:=\min _{X} f$ and $\sigma>0$, then

$$
f(x)-\alpha \geq \sigma d(x,[f \leq \alpha]) \quad \text { for all } x \in X
$$

if and only if

$$
\partial f^{*}\left(x^{*}\right) \subset \partial f^{*}(0) \text { for all } x^{*} \in X^{*} \text { such that }\left\|x^{*}\right\|_{*}<\sigma,
$$

where $f^{*}$ denotes the Fenchel conjugate of $f$. In fact, as $x \in \partial f^{*}\left(x^{*}\right)$ if and only if $x^{*} \in \partial f(x)$, the latter condition is clearly equivalent to $\inf _{x \in[f>\alpha]} d_{*}(0, \partial f(x)) \geq \sigma$. The result was stated under this form in [18] (Props. 3.1 and 7.1) (more recently, see also [28] (Cor. 2.2), or the book [29] by the same author). Recall that in Theorem 3.1, the fact that $\operatorname{argmin} f \neq \emptyset$ is a consequence of the fact that $\inf _{x \in[f>\alpha]} d_{*}(0, \partial f(x))>0$, if $\alpha=\inf _{X} f>-\infty$.

(c) Let the Banach space $X$ be reflexive and $\alpha \in \mathbb{R}$ be such that $[f \leq \alpha] \neq \emptyset$. Let then $\alpha<\beta \leq+\infty$, $x \in[f>\alpha] \cap \operatorname{dom} f, y \in[f \leq \alpha]$ be such that $\|x-y\|=d(x,[f \leq \alpha])$, so that $f(y)=\alpha$, and let $\bar{\lambda} \in] 0,1]$ be such that $f(y+\lambda(x-y)) \in] \alpha, \beta[$ for $\lambda \in] 0, \bar{\lambda}]$. Then, for every such $\lambda$, we have:

$$
\sigma_{\alpha, \beta}(f) \leq \frac{f(y+\lambda(x-y))-\alpha}{d(y+\lambda(x-y),[f \leq \alpha])}=\frac{f(y+\lambda(x-y))-f(y)}{\lambda\|x-y\|} \leq \frac{f(x)-f(y)}{\|x-y\|}=\frac{f(x)-\alpha}{d(x,[f \leq \alpha])},
$$

and thus (since, obviously, $\sigma_{\alpha}(f) \leq \sigma_{\alpha, \beta}(f)$ ):

$$
\sigma_{\alpha, \beta}(f)=\sigma_{\alpha}(f)=\inf \left\{\frac{f^{\prime}\left(y ; J^{-1}\left(y^{*}\right)\right)}{\left\|y^{*}\right\|_{*}}: f(y)=\alpha, y^{*} \in N_{[f \leq \alpha]}(y) \backslash\{0\}\right\},
$$

where $J: X \rightarrow X^{*}$ denotes the duality mapping, and $N_{[f \leq \alpha]}(y)$ denotes the usual normal cone to the convex set $[f \leq \alpha]$ at the point $y$. This result - in fact, the second equality - was established by Lewis and Pang in [19] (Th. 1, Cor. 1), in the case $X=\mathbb{R}^{n}$ (see also [28], (Th. 2.1, Prop. 3.5) in the more general case). Observe that 
such characterization of the existence of a global error bound depends only on the definitions (the variational principle is not needed for this).

(d) Finally, and following Auslender and Crouzeix [1] (see also [2]), we recall that the convex lower semicontinuous function $f: X \rightarrow \mathbb{R} \cup\{+\infty\}$ has a good asymptotical behaviour (GAB, for short) if for any sequence $\left(x_{n}\right) \subset X$ we have:

$$
d_{*}\left(0, \partial f\left(x_{n}\right)\right) \rightarrow 0 \Longrightarrow f\left(x_{n}\right) \rightarrow \inf _{X} f .
$$

This notion naturally provides a verifiable sufficient condition for the existence of global error bounds for $f$. Namely, it readily follows from the first part of Theorem 3.1 (with $\beta=+\infty$ ) that $f$ has a good asymptotical behaviour if and only if $\sigma_{\alpha}(f)>0$ for any $\alpha>\inf _{X} f$ (recall that " $\alpha>\inf _{X} f$ " reads: " $f$ satisfies the Slater qualification condition at level $\alpha$ ", in the terminology of mathematical programming). The condition GAB was used in [6] in order to derive a Hoffman-type estimate in semidefinite optimization.

Combining Theorem 2.1, Propositions 3.1 and 3.2, we obtain the following, yielding a sufficient condition for a global error bound.

Theorem 3.2. Let $X$ be a Banach space, $f: X \rightarrow \mathbb{R} \cup\{+\infty\}$ be a convex, lower semicontinuous function, and $\alpha \in \mathbb{R}$ such that $[f \leq \alpha] \neq \emptyset$. Then:

$$
\inf _{x \in[f>\alpha]} \frac{f(x)-\alpha}{d(x,[f \leq \alpha])} \geq \inf _{x \in[f=\alpha]} d_{*}(0, \partial f(x)) .
$$

Of course, we can choose in Theorems 3.1 and 3.2 another estimate of the strong slope than $d_{*}(0, \partial f)$, according to Proposition 3.1. For example, we have the following characterization of the existence of a global error bound for convex inequalities, which we state in a "reversed" form, for the sake of comparison with an earlier result in the literature.

Theorem 3.3. Let $X$ be a Banach space, $f: X \rightarrow \mathbb{R} \cup\{+\infty\}$ be a proper, convex, and lower semicontinuous function, and $\alpha \in \mathbb{R}, \beta \in \mathbb{R} \cup\{+\infty\}$ with $\alpha<\beta$. Then:

$$
\sup _{x \in[\alpha<f<\beta]} \frac{d(x,[f \leq \alpha])}{f(x)-\alpha}=\sup _{x \in[\alpha<f<\beta]} \inf _{f(z)<f(x)} \frac{\|x-z\|}{f(x)-f(z)} .
$$

Moreover, if $[f<\beta] \neq \emptyset$, then:

$$
\sup _{x \in[f>\alpha]} \frac{d(x,[f \leq \alpha])}{f(x)-\alpha}=\sup _{x \in[\alpha<f<\beta]} \inf _{f(z)<f(x)} \frac{\|x-z\|}{f(x)-f(z)} .
$$

Remark 3.3. (a) The above result is obtained in [26] (Th. 8) (expressed in a different way), under the additional assumption that $X$ is reflexive. This restriction is probably due to the method employed in [26], which makes use, in particular, of the result described in Remark 2.2(b) (and not of the precise informations given by the strong slope).

(b) Following Mangasarian [20], we say that the proper, convex, lower semicontinuous function $f: X \rightarrow$ $\mathbb{R} \cup\{+\infty\}$ ( $X$ a Banach space) satisfies the strong Slater qualification condition at the level $\gamma \in \mathbb{R}$ if

$$
\sup _{x \in[f=\gamma]} \inf _{f(z)<\gamma} \frac{\|x-z\|}{\gamma-f(z)}<+\infty .
$$

According to Proposition 3.1, this is equivalent to say that $\inf _{[f=\gamma]}|\nabla f|>0$ (which is true, in particular, if $[f=\gamma]=\emptyset)$. We also say that $f$ satisfies the strong Slater qualification condition on an interval $] \alpha, \beta[$ 
$(-\infty<\alpha<\beta \leq+\infty)$ if

$$
\sup _{\alpha<\gamma<\beta} \sup _{x \in[f=\gamma]} \inf _{f(z)<\gamma} \frac{\|x-z\|}{\gamma-f(z)}<+\infty,
$$

which is equivalent to $\inf _{[\alpha<f<\beta]}|\nabla f|>0$. Theorem 3.3 says that $f$ has a global error bound between the levels $\alpha$ and $\beta$ if and only if $f$ satisfies the strong Slater qualification condition on $] \alpha$, $\beta[$, and that if $[f<\beta] \neq \emptyset$, the latter condition is equivalent to $f$ having a global error bound above the level $\alpha$.

Moreover, it follows from Theorem 3.2 that if $[f \leq \alpha] \neq \emptyset$ and if $f$ satisfies the strong Slater qualification condition at the level $\alpha$, then $f$ has a global error bound above the level $\alpha$. This considerably extends the main result of $[20]$, where $X=\mathbb{R}^{n}$ and $f$ is finite-valued (hence continuous).

\section{Subdifferential operators}

As in the previous section, we consider here a Banach space $X$ endowed with a norm $\|\cdot\|$, with topological dual $X^{*}, d_{*}$ denoting the metric associated with the norm of $X^{*}$.

We further consider an "abstract" subdifferential operator $\partial$, which associates to any lower semicontinuous function $f: X \rightarrow \mathbb{R} \cup\{+\infty\}$, and any point $x \in X$, a subset $\partial f(x)$ of the (topological) dual $X^{*}$ of $X$, in such a way that $\partial f(x)=\emptyset$ if $x \notin \operatorname{dom} f$, and the following two properties are satisfied:

(P1) if $f$ is convex, then $\partial f(x)=\left\{x^{*} \in X^{*}: f(y) \geq f(x)+\left\langle x^{*}, y-x\right\rangle \forall y \in X\right\}$;

(P2) if $g: X \rightarrow \mathbb{R}$ is convex and Lipschitz continuous, and if $\bar{x} \in \operatorname{dom} f$ is a local minimum point of $f+g$ then, for every $\varepsilon>0$ there exist $x, y \in X, x^{*} \in \partial f(x)$, and $y^{*} \in \partial g(y)$ such that

$$
\|x-\bar{x}\| \leq \varepsilon,\|y-\bar{x}\| \leq \varepsilon, \quad f(x) \leq f(\bar{x})+\varepsilon, \text { and }\left\|x^{*}+y^{*}\right\|_{*} \leq \varepsilon
$$

Proposition 4.1. Let $X$ be a Banach space and $\partial$ be a subdifferential operator such that properties (P1) and (P2) are satisfied. Then, for every lower semicontinuous function $f: X \rightarrow \mathbb{R} \cup\{+\infty\}$ and every $x \in X$, we have:

$$
|\nabla f|(x) \geq \liminf _{(y, f(y)) \rightarrow(x, f(x))} d_{*}(0, \partial f(y))
$$

Proof. Let $x \in X$. We may assume that $x$ is not a local minimum point of $f$ (for, otherwise, the result readily follows from (P2) with $g \equiv 0$ ), and that $|\nabla f|(x)<+\infty$ (so that $x \in \operatorname{dom} f)$. Let $\sigma>|\nabla f|(x)$ and $\varepsilon>0$, let then $r>0$ such that

$$
f(x) \leq f(y)+\sigma\|y-x\| \quad \text { for every } y \in B_{r}(x),
$$

so that the function $f+\sigma\|\cdot-x\|$ attains a finite local minimum at $x$. From property (P2), we thus find $y, z \in X$ and $y^{*} \in \partial f(y), z^{*} \in \partial(\sigma\|\cdot-x\|)(z)$ such that

$$
\|y-x\| \leq \varepsilon, \quad f(y) \leq f(x)+\varepsilon, \quad \text { and } \quad\left\|y^{*}+z^{*}\right\|_{*} \leq \varepsilon
$$

From property $(\mathrm{P} 1),\left\|z^{*}\right\|_{*} \leq \sigma$, so that $\left\|y^{*}\right\|_{*} \leq \sigma+\varepsilon$, and the conclusion follows since $\varepsilon>0$ is arbitrary, taking into account the lower semicontinuity of $f$.

As an immediate consequence of Theorem 2.1 and Proposition 4.1 we thus have:

Corollary 4.1. Let $X$ be a Banach space and $\partial$ be a subdifferential operator such that (P1) and (P2) hold, $f: X \rightarrow \mathbb{R} \cup\{+\infty\}$ be a lower semicontinuous function, and $\alpha \in \mathbb{R}, \beta \in \mathbb{R} \cup\{+\infty\}$ with $\alpha<\beta$. Then:

$$
\inf _{\alpha \leq \gamma<\beta} \sigma_{\gamma, \beta}(f) \geq \inf _{x \in[\alpha<f<\beta]} d_{*}(0, \partial f(x))
$$

Remark 4.1. (a) Consideration of axiomatically defined subdifferentials, and of so-called fuzzy calculus rules such as (P2), were initiated by Ioffe in the 1980's, and have since then been widely developed and used. As is well known, and unlike property (P1), property (P2) is usually not an intrinsic property of a specific subdifferential 
operator, but a property of the pair $(X, \partial)$, and that such property follows, for specific pairs, either from Ekeland's variational principle, or from one of its "smooth" variants due to Borwein and Preiss, and to Deville, Godefroy and Zizler (see, e.g., [5, 15,17] for examples and more details). In [5], we introduced an abstract notion of variational pair, in order to derive results of the type of Theorem 2.1 and of Corollary 4.1 as special cases. We now believe that it is preferable to derive results like Corollary 4.1, via Proposition 4.1, from Theorem 2.1 which is a truly general, metric result, providing a necessary and sufficient condition for the existence of global error bounds. This was observed by Ioffe after he became aware of the paper [5], and exploited in [16] in the context of metric regularity theory: see Section 5 .

(b) Writing property (P2) with $\varepsilon=0$, we obtain the following (stronger) exact sum rule:

$(\widehat{\mathrm{P} 2})$ if $g: X \rightarrow \mathbb{R}$ is convex and Lipschitz continuous, and if $\bar{x} \in \operatorname{dom} f$ is a local minimum point of $f+g$ then, $0 \in \partial f(x)+\partial g(x)$.

Then, arguing as in Proposition 4.1 yields, of course, the stronger conclusion:

$$
|\nabla f|(x) \geq d_{*}(0, \partial f(x)) \quad \text { for every } x \in X
$$

See also Remark 5.3(a) below, concerning the reverse inequality, for some specific subdifferential operator.

(c) The strong slope can also be compared, just using the definitions, with various notions of directional derivative. For example, we have:

$$
\|u\||\nabla f|(x) \geq-f^{\prime}(x ; u) \geq-f^{-}(x ; u) \quad \text { for all } x, u \in X
$$

where

$$
f^{\prime}(x ; u):=\liminf _{\substack{t \\ v \rightarrow u}} \frac{f(x+t v)-f(x)}{t}, \quad f^{-}(x ; u):=\liminf _{t \searrow 0} \frac{f(x+t u)-f(x)}{t}
$$

are respectively the (lower) contingent and Dini derivative of $f$ at $x$ in the direction $u$.

This shows, for example, that Theorem 2.1 (recall (6)) yields, as a special case, an extension of [23] (Th. 2.5) (see also [26], (Th. 4)).

(d) From the point of view of applications, and when dealing with functions $f$ which are not convex, a result like Corollary 4.1 may not be so appropriate or meaningful: one would rather need a local version of the result - what we shall deal with in the coming and last section of this paper.

\section{LOCAL RESULTS AND A REMARK ON METRIC REGULARITY}

In this section, we give two results dealing with sufficient conditions for what might be called a local error bound for $f: X \rightarrow \mathbb{R} \cup\{+\infty\}$. The first one is indeed a direct extension of the sufficient condition given by Theorem 2.1, which will be applied (as an example) to the characterization (taking Prop. 2.1 into account) of so-called weak sharp local minima; the second one is a variant which will be specifically applied to the characterization of the local metric regularity of a closed multifunction between complete metric spaces.

Theorem 5.1. Let $X$ be complete, $f: X \rightarrow \mathbb{R} \cup\{+\infty\}$ be a lower semicontinuous function, $\alpha \in \mathbb{R}, \beta \in$ $\mathbb{R} \cup\{+\infty\}$ with $\alpha<\beta, U \subset[f \leq \alpha]$, and $\rho>0$. Then:

$$
\inf _{\alpha \leq \gamma<\beta} \inf _{x \in \bar{B}_{\rho}(U) \cap[\gamma<f<\beta]} \frac{f(x)-\gamma}{d(x,[f \leq \gamma])} \geq \inf _{B_{2 \rho}(U) \cap[\alpha<f<\beta]}|\nabla f| .
$$

Proof. Let $\gamma \in[\alpha, \beta[$, we shall show that

$$
\inf _{x \in \bar{B}_{\rho}(U) \cap[\gamma<f<\beta]} \frac{f(x)-\gamma}{d(x,[f \leq \gamma])} \geq \inf _{B_{2 \rho}(U) \cap[\gamma<f<\beta]}|\nabla f|,
$$


which clearly yields the conclusion of the theorem. We may assume that the left-hand side of the inequality is finite, so that $\bar{B}_{\rho}(U) \cap[\gamma<f<\beta]$, hence also $[f \leq \gamma]$, is nonempty. As in the proof of Theorem 2.1, consider a real number $\sigma>0$ and $\bar{x} \in \bar{B}_{\rho}(U) \cap[\gamma<f<\beta]$ with

$$
f(\bar{x})-\gamma<\sigma d(\bar{x},[f \leq \gamma]) .
$$

Let $0<r:=d(\bar{x},[f \leq \gamma]) \leq d(\bar{x}, U) \leq \rho$, and $g:=(f-\gamma)^{+} \geq 0$, so that

$$
g(\bar{x})<\inf _{\bar{B}_{r}(\bar{x})} g+\sigma r .
$$

Applying Corollary 2.1, we find $x \in B_{r}(\bar{x})$ with $g(x) \leq g(\bar{x})$ and $|\nabla g|(x)<\sigma$. Then, $d(x, U) \leq d(x, \bar{x})+$ $d(\bar{x}, U)<2 \rho$, while $f(x)>\gamma$, so that $f(x)=g(x)+\gamma \leq g(\bar{x})+\gamma=f(\bar{x})<\beta$ and $|\nabla f|(x)=|\nabla g|(x)<\sigma$, establishing (9).

Remark 5.1. Let $X$ be complete, $f: X \rightarrow \mathbb{R} \cup\{+\infty\}$ be lower semicontinuous, $\bar{x} \in \operatorname{dom} f$, and $\rho>0$ such that $f(\bar{x}) \leq f(x)$ for all $x \in \bar{B}_{2 \rho}(\bar{x})$. For $\varepsilon \geq 0$, let $S_{\varepsilon}:=[f \leq f(\bar{x})+\varepsilon]$. Theorem 5.1 tells us that if

$$
\sigma:=\inf _{B_{2 \rho}(\bar{x}) \backslash S_{0}}|\nabla f|(x)>0,
$$

then:

In particular:

$$
f(x)-(f(\bar{x})+\varepsilon) \geq \sigma d\left(x, S_{\varepsilon}\right) \quad \forall x \in \bar{B}_{\rho}(\bar{x}) \backslash S_{\varepsilon}, \forall \varepsilon \geq 0 .
$$

$$
f(x) \geq f(\bar{x})+\sigma d(x, S) \quad \forall x \in \bar{B}_{\rho}(\bar{x}),
$$

where $S:=\left\{x \in \bar{B}_{\rho}(\bar{x}): f(x)=f(\bar{x})\right\}$. Conversely, if (10) holds true, then

$$
\inf _{B_{\rho}(\bar{x}) \backslash S_{0}}|\nabla f| \geq \sigma,
$$

thanks to Proposition 2.1, which indeed provides a converse statement to Theorem 5.1. As in [25], and following the terminology introduced by Burke and Ferris [7], we say that $\bar{x}$ is a weak sharp local minimum point of $f$ if (10) is satisfied (for some $\sigma>0$ ), and this remark thus gives a characterization of such weak sharp local minima. Compare with the results of [25], in particular, [25] (Th. 5.2), giving a sufficient condition, in terms of the so-called Mordukhovich subdifferential, for (11) to be satisfied, for a continuous $f: \mathbb{R}^{n} \rightarrow \mathbb{R}$.

Of course, in the convex case, local becomes global, and the characterization of weak sharp minima becomes even sharper, as given in the results of Section 3 .

Theorem 5.2. Let $X$ be complete, $f: X \rightarrow \mathbb{R} \cup\{+\infty\}$ be a lower semicontinuous function, $U$ be a subset of $X, \alpha \in \mathbb{R}$, and $\sigma, \rho>0$. Assume that $U \cap[f<\alpha+\sigma \rho] \neq \emptyset$ and that

$$
\inf _{B_{\rho}(U) \cap[\alpha<f<\alpha+\sigma \rho]}|\nabla f| \geq \sigma .
$$

Then, $[f \leq \alpha] \neq \emptyset$, and:

$$
\inf _{x \in U \cap[\alpha<f<\alpha+\sigma \rho]} \frac{f(x)-\alpha}{d(x,[f \leq \alpha])} \geq \sigma .
$$

Proof. The fact that $[f \leq \alpha] \neq \emptyset$ is established in Proposition 2.3. We may now assume that $U \cap[\alpha<f<\alpha+\sigma \rho] \neq$ $\emptyset$. Assume further, for a contradiction, that for some $\bar{x} \in U \cap[\alpha<f<\alpha+\sigma \rho]$ we have:

$$
f(\bar{x})-\alpha<\sigma d(\bar{x},[f \leq \alpha]) .
$$


Let $0<r:=\min \{d(\bar{x},[f \leq \alpha]), \rho\}$, and $g:=(f-\alpha)^{+}$, so that

$$
g(\bar{x})<\inf _{\bar{B}_{r}(\bar{x})} g+\sigma r .
$$

Applying Corollary 2.1, we find $x \in B_{r}(\bar{x})$ with $g(x) \leq g(\bar{x})$ and $|\nabla g|(x)<\sigma$. Then, $x \in B_{\rho}(U) \cap[\alpha<f<\alpha+\sigma \rho]$ and $|\nabla f|(x)=|\nabla g|(x)<\sigma$ : a contradiction.

Remark 5.2. Thanks to Proposition 4.1, Theorems 5.1 and 5.2 contain, as a special case, [27] (Th. 2.2), where $X$ is a Banach space, $U$ is a singleton, and the sufficient condition is given in terms of a subdifferential operator. We observe again that our proofs are also much simpler, which helps understand such results.

We shall need the following definition, see Remark 5.3(b) below for comments.

Definition 5.1. For $z \in X$, let $d_{z}: X \rightarrow \mathbb{R}$ be defined by $d_{z}(x):=d(z, x)$. We say that the metric space $X$ is coherent at $z \in X$ if

$$
\left|\nabla d_{z}\right|(x)=1 \quad \text { for every } x \neq z .
$$

If this is true for every $z \in X$, we just say that $X$ is coherent.

Clearly, if $X$ is a convex subset of a normed vector space, then $X$ (with the metric associated with the norm) is coherent. In general, since $d_{z}$ is 1-Lipschitzian, so that $\left|\nabla d_{z}\right| \leq 1$, the fact that $X$ is coherent at $z$ is equivalent to:

$$
d(z, x)-\gamma \geq d\left(x, \bar{B}_{\gamma}(z)\right) \quad \forall x \in X \backslash \bar{B}_{\gamma}(z), \forall \gamma \geq 0,
$$

according to Theorem 2.1.

We now consider two metric spaces $X$ and $Y$ (we shall use the same notation $d$ for both metrics), and, for $\delta>0$, the product space $X \times Y$ as endowed with the metric:

$$
d_{\delta}\left((x, y),\left(x^{\prime}, y^{\prime}\right)\right):=\max \left\{d\left(x, x^{\prime}\right), \delta d\left(y^{\prime}, y^{\prime}\right)\right\} .
$$

Accordingly, if $f: X \times Y \rightarrow \mathbb{R} \cup\{+\infty\}$ is a lower semicontinuous function, we shall let $\left|\nabla_{\delta} f\right|$ denote the strong slope of $f$ with respect to the metric $d_{\delta}$.

We further consider a multifunction $F \subset X \times Y$ : for $x \in X$ and $y \in Y$ we respectively set:

$$
F(x):=\{y \in Y:(x, y) \in F\}, \quad F^{-1}(y):=\{x \in X:(x, y) \in F\} .
$$

For $z \in Y$, we define a function $f_{z}: X \times Y \rightarrow \mathbb{R} \cup\{+\infty\}$ by:

$$
f_{z}(x, y):= \begin{cases}d(z, y) & \text { if }(x, y) \in F \\ +\infty & \text { otherwise }\end{cases}
$$

so that $f_{z}$ is lower semicontinuous if (and only if) $F$ is closed ( $F$ is a closed-graph multifunction). Moreover, for every $\gamma \geq 0$, we have:

$$
\left[f_{z} \leq \gamma\right]=\bigcup_{y \in \bar{B}_{\gamma}(z)}\left(F^{-1}(y) \times\{y\}\right)
$$

(where, of course, $F^{-1}(y) \times\{y\}=\emptyset$ if $F^{-1}(y)=\emptyset$ ).

Theorem 5.3. Let $X$ and $Y$ be complete, $F \subset X \times Y$ be a closed multifunction, $(\bar{x}, \bar{y}) \in F, V$ and $W$ be neighborhoods of $\bar{x}$ and $\bar{y}$, respectively, $\sigma>0$, and $0<\delta \leq 1 / \sigma$.

(a) Assume that:

$$
\left|\nabla_{\delta} f_{z}\right|(x, y) \geq \sigma \quad \forall(x, y, z) \in V \times W \times W, y \neq z .
$$


Then, there exists $\varepsilon>0$ such that:

$$
d(z, F(x)) \geq \sigma d\left(x, F^{-1}(z)\right) \quad \forall(x, z) \in B_{\varepsilon}(\bar{x}) \times B_{\varepsilon}(\bar{y}) .
$$

(b) Conversely, assume that $Y$ is coherent in a neighborhood of $\bar{y}$, and that:

$$
d(z, F(x)) \geq \sigma d\left(x, F^{-1}(z)\right) \quad \forall(x, z) \in V \times W .
$$

Then, there exists $r>0$ such that

$$
\left|\nabla_{\delta} f_{z}\right|(x, y) \geq \sigma \quad \forall(x, y, z) \in B_{r}(\bar{x}) \times B_{r}(\bar{y}) \times B_{r}(\bar{y}), y \neq z .
$$

Proof. (a) Let $\rho>0$ be such that $B_{2 \rho}(\bar{x}, \bar{y}) \subset V \times W$, and let $z \in B_{\sigma \rho}(\bar{y}) \subset W$. Applying Theorem 5.2 to $f_{z}$ with $U:=B_{\rho}(\bar{x}, \bar{y}), \alpha:=0$, and the given $\sigma$ and $\rho$, since $(\bar{x}, \bar{y}) \in U \cap\left[f_{z}<\sigma \rho\right]$ and $\left|\nabla_{\delta} f_{z}\right|(x, y) \geq \sigma$ for every $(x, y) \in\left[f_{z}>0\right] \cap B_{\rho}(U)$, we obtain:

$$
f_{z}(x, y) \geq \sigma d_{\delta}\left((x, y),\left[f_{z} \leq 0\right]\right) \quad \forall(x, y) \in\left[f_{z}<\sigma \rho\right] \cap B_{\rho}(\bar{x}, \bar{y}) .
$$

Let $0<r \leq \rho$ be such that $B_{r}(\bar{x}, \bar{y}) \cap F \subset\left[f_{z}<\sigma \rho\right]$ for every $z \in B_{\sigma r}(\bar{y})$. Then, taking the definitions of $f_{z}$ and of $d_{\delta}$, the fact that $\sigma \delta \leq 1$, and (13), into account, (15) yields:

$$
d(z, y) \geq \sigma d\left(x, F^{-1}(z)\right) \quad \forall(x, y) \in B_{r}(\bar{x}, \bar{y}) \cap F, \forall z \in B_{\sigma r}(\bar{y}),
$$

and in particular:

$$
d(z, \bar{y}) \geq \sigma d\left(\bar{x}, F^{-1}(z)\right) \quad \forall z \in B_{\sigma r}(\bar{y})
$$

Assume now that the conclusion does not hold. Then, there exist sequences $\left(x_{n}, y_{n}\right) \subset F$ and $\left(z_{n}\right) \subset B_{\sigma r}(\bar{y})$ such that $d\left(x_{n}, \bar{x}\right) \rightarrow 0, d\left(z_{n}, \bar{y}\right) \rightarrow 0$, and:

$$
d\left(z_{n}, y_{n}\right)<\sigma d\left(x_{n}, F^{-1}\left(z_{n}\right)\right)
$$

Taking (17) into account yields:

$$
d\left(y_{n}, \bar{y}\right)-d\left(z_{n}, \bar{y}\right)<\sigma d\left(x_{n}, \bar{x}\right)+\sigma d\left(\bar{x}, F^{-1}\left(z_{n}\right)\right) \leq \sigma d\left(x_{n}, \bar{x}\right)+d\left(z_{n}, \bar{y}\right),
$$

which shows that $d\left(y_{n}, \bar{y}\right) \rightarrow 0$, so that (18) contradicts (16) for large $n$.

(b) Let $r>0$ be such that $B_{r}(\bar{x}) \subset V, B_{3 r}(\bar{y}) \subset W$, and $Y$ is coherent in $B_{r}(\bar{y})$. Let $z \in B_{r}(\bar{y})$ be fixed, let then $\gamma \geq 0$ and $(x, y) \in F \cap\left(B_{r}(\bar{x}) \times B_{r}(\bar{y})\right) \cap\left[f_{z}>\gamma\right]$, so that $\gamma<d(z, y) \leq 2 r$. Then, for every $y^{\prime} \in \bar{B}_{\gamma}(z) \subset V$, we have:

(in particular, $F^{-1}\left(y^{\prime}\right) \neq \emptyset$ for every such $\left.y^{\prime}\right)$, hence:

$$
d\left(y^{\prime}, y\right) \geq \sigma d\left(x, F^{-1}\left(y^{\prime}\right)\right)
$$

$$
d\left(y^{\prime}, y\right) \geq \sigma d_{\delta}\left((x, y), F^{-1}\left(y^{\prime}\right) \times\left\{y^{\prime}\right\}\right),
$$

and finally, since $y^{\prime}$ is arbitrary in $\bar{B}_{\gamma}(z)$ :

$$
d\left(y, \bar{B}_{\gamma}(z)\right) \geq \sigma d_{\delta}\left((x, y),\left[f_{z} \leq \gamma\right]\right) .
$$

According to (12), we have:

$$
f_{z}(x, y)-\gamma \geq \sigma d_{\delta}\left((x, y),\left[f_{z} \leq \gamma\right]\right) .
$$


Letting $U:=B_{r}(\bar{x}) \times B_{r}(\bar{y})$, we thus have:

$$
\inf _{\gamma>0} \inf _{(x, y) \in U \cap\left[f_{z}>\gamma\right]} \frac{f_{z}(x, y)-\gamma}{d_{\delta}\left((x, y),\left[f_{z} \leq \gamma\right]\right)} \geq \sigma,
$$

and the conclusion follows from Proposition 2.1 applied to $f_{z}$ with $\alpha:=0$ and $\beta:=+\infty$, since $z$ is arbitrary in $B_{r}(\bar{y})$.

Remark 5.3. (a) According to the terminology in use, condition (14) in Theorem 5.3 means that the multifunction $F$ is metrically regular (with constant $\sigma$ ) near the point $(\bar{x}, \bar{y})$. Part (a) of the result is an abstract version of a number of results dealing with sufficient conditions for local metric regularity, in the context of Banach rather than metric spaces, going back to Ioffe (see $[13,14]$ ), and extending the classical theorem of Lusternik and Graves. A rather general Banach space setting is the following: let $X$ and $Y$ be Asplund spaces, let $F \subset X \times Y$ be a closed multifunction, and $(\bar{x}, \bar{y}) \in F$. Assume that:

$$
\liminf _{(x, y) \rightarrow(\bar{x}, \bar{y})} d_{*}\left(0, D_{\mathcal{F}}^{*} F(x, y)\left(S_{Y^{*}}\right)\right)>\sigma>0,
$$

where $D_{\mathcal{F}}^{*} F$ stands for the Fréchet coderivative of $F$ in the sense of Mordukhovich $[21,22],(x, y) \stackrel{F}{\rightarrow}(\bar{x}, \bar{y})$ means that $(x, y)$ converges to $(\bar{x}, \bar{y})$ in $F$, and $S_{Y^{*}}$ denotes the unit sphere in the dual space $Y^{*}$. Then, we can use Theorem 5.3(a), choosing $\delta:=\min \left\{1, \frac{1}{\sigma}\right\}$, to deduce that (14) holds: see [5], especially Corollary 5.7 therein, and use Proposition 4.1 with $\partial:=\partial_{\mathcal{F}}$, the Fréchet subdifferential (indeed, property (P2) holds for $\partial:=\partial_{\mathcal{F}}$ if and only if the Banach space $X$ is Asplund, according to [11]). Conversely, since for any Banach space $X$ and any lower semicontinuous function $f: X \rightarrow \mathbb{R} \cup\{+\infty\}$, we have:

$$
|\nabla f|(x) \leq d_{*}\left(0, \partial_{\mathcal{F}} f(x)\right)
$$

(as readily follows from the definitions), Theorem 5.3(b) allows to deduce that (14) implies (19) (with " $\geq \sigma$ "). We thus recover as special cases the main results of [22].

(b) In the line of the approach of [5], Ioffe established in [16] (Th. 2) a characterization of local metric regularity for closed multifunctions in metric spaces, using the strong slope. In order to obtain the necessary condition for metric regularity, it is assumed in [16] that the metric space $Y$ satisfies the following geodesic property: for every $y_{1}, y_{2} \in Y, y_{1} \neq y_{2}$, and for every $\varepsilon>0$, there exists $y \in Y$ such that:

$$
d\left(y, y_{i}\right) \leq \frac{d\left(y_{1}, y_{2}\right)}{2}+\varepsilon, \quad i=1,2 .
$$

This property is in fact equivalent to $Y$ being coherent. Indeed, assuming that the geodesic property holds, considering $z, x \in Y, x \neq z$, and a sequence $\left.\left(\varepsilon_{n}\right) \in\right] 0, d(z, x)$ [ such that $2^{n} \varepsilon_{n} \rightarrow 0$, it is not difficult to construct, recursively, a sequence $\left(x_{n}\right) \subset Y$ such that

$$
d\left(x_{n}, x\right) \leq \frac{1}{2^{n}} d(z, x)+\varepsilon_{n}, \quad d\left(x_{n}, z\right) \leq\left(1-\frac{1}{2^{n}}\right) d(z, x)+\varepsilon_{n},
$$

so that $x_{n} \rightarrow x, d\left(z, x_{n}\right)<d(z, x)$, and:

$$
\left|\nabla d_{z}\right|(x) \geq \limsup _{n \rightarrow \infty} \frac{d(z, x)-d\left(z, x_{n}\right)}{d\left(x, x_{n}\right)} \geq \lim _{n \rightarrow \infty} \frac{d(z, x)-2^{n} \varepsilon_{n}}{d(z, x)+2^{n} \varepsilon_{n}}=1,
$$

showing that $Y$ is coherent at $z$. Conversely, considering $y_{1}, y_{2} \in Y$ with $y_{1} \neq y_{2}$, and $\varepsilon>0$, since $Y$ is coherent 
at $y_{1}$ we deduce from $(12)$ that

$$
d\left(y_{1}, y_{2}\right)-\gamma \geq d\left(y_{2}, \bar{B}_{\gamma}\left(y_{1}\right)\right)
$$

where $\gamma:=\frac{1}{2} d\left(y_{1}, y_{2}\right)$. We thus see that $(20)$ is satisfied by any $y \in \bar{B}_{\gamma}\left(y_{1}\right)$ such that $d\left(y_{2}, y\right) \leq d\left(y_{2}, \bar{B}_{\gamma}\left(y_{1}\right)\right)+\varepsilon$.

Thus, Theorem 5.3 is similar to [16] (Th. 2) but, in our opinion, the notion of coherence of a metric space is more in line with the general approach developed in this paper than the geodesic property, and provides a better insight into the above result.

Acknowledgements. We thank one of the anonymous referees for a relevant observation.

\section{REFERENCES}

[1] A. Auslender and J.-P. Crouzeix, Well behaved asymptotical convex functions. Ann. Inst. H. Poincaré, Anal. Non Linéaire 6 (1989) 101-121.

[2] A. Auslender, R. Cominetti and J.-P. Crouzeix, Convex functions with unbounded level sets. SIAM J. Optim. 3 (1993) 669-687.

[3] A. Auslender and M. Teboulle, Asymptotic Cones and Functions in Optimization and Variational Inequalities. Springer Monogr. Math. (2003).

[4] D. Azé and J.-N. Corvellec, On the sensitivity analysis of Hoffman constants for systems of linear inequalities. SIAM J. Optim. 12 (2002) 913-927.

[5] D. Azé, J.-N. Corvellec and R.E. Lucchetti, Variational pairs and applications to stability in nonsmooth analysis. Nonlinear Anal. 49 (2002) 643-670.

[6] D. Azé and J.-B. Hiriart-Urruty, Optimal Hoffman-type estimates in eigenvalue and semidefinite inequality constraints. $J$. Global Optim. 24 (2002) 133-147.

[7] J.V. Burke and M.C. Ferris, Weak sharp minima in mathematical programming. SIAM J. Control Optim. 31 (1993) 1340-1359.

[8] O. Cornejo, A. Jourani and C. Zălinescu, Conditioning and upper-Lipschitz inverse subdifferentials in nonsmooth optimization problems. J. Optim. Theory Appl. 95 (1997) 127-148.

[9] E. De Giorgi, A. Marino and M. Tosques, Problemi di evoluzione in spazi metrici e curve di massima pendenza (Evolution problems in metric spaces and curves of maximal slope). Atti Accad. Naz. Lincei Rend. Cl. Sci. Fis. Mat. Natur. 68 (1980) 180-187.

[10] I. Ekeland, Nonconvex minimization problems. Bull. Amer. Math. Soc. 1 (1979) 443-474.

[11] M. Fabian, Subdifferentiability and trustworthiness in the light of the new variational principle of Borwein and Preiss. Acta Univ. Carolin. 30 (1989) 51-56.

[12] A.J. Hoffman, On approximate solutions of systems of linear inequalities. J. Res. Nat. Bur. Stand. 49 (1952) 263-265.

[13] A. Ioffe, Regular points of Lipschitz functions. Trans. Amer. Math. Soc. 251 (1979) 61-69.

[14] A. Ioffe, On the local surjection property. Nonlinear Anal. 11 (1987) 565-592.

[15] A. Ioffe, Variational methods in local and global non-smooth analysis, in Nonlinear Analysis, Differential Equations and Control, Montréal, 1998, F.H. Clarke and R.J. Stern Eds., Kluwer, Dordrecht, NATO Sc. Ser., C 528 (1999) 447-502.

[16] A. Ioffe, Towards metric theory of metric regularity, in Approximation, Optimization and Mathematical Economics, Guadeloupe, 1999, M. Lassonde Ed., Physica-Verlag, Heidelberg (2001) 165-176.

[17] M. Lassonde, First order rules for nonsmooth constrained optimization. Nonlinear Anal. 44 (2001) 1031-1056.

[18] B. Lemaire, Well-posedness, conditioning and regularization of minimization, inclusion and fixed-point problems. Pliska Stud. Math. Bulgar. 12 (1998) 71-84.

[19] A.S. Lewis and J.S. Pang, Error bounds for convex inequality systems, in Generalized Convexity, Generalized Monotonicity: Recent Results, Marseille, 1996, J.-P. Crouzeix et al. Eds., Kluwer, Dordrecht, Nonconvex Optim. Appl. 27 (1998).

[20] O.L. Mangasarian, Error bounds for nondifferentiable convex inequalities under a strong Slater constraint qualification. Math. Program. 83 (1998) 187-194.

[21] B.S. Mordukhovich, Metric approximations and necessary optimality conditions for general classes of nonsmooth extremal problems. Soviet Math. Dokl. 22 (1980) 526-530.

[22] B.S. Mordukhovich and Y. Shao, Differential characterizations of covering, metric regularity and Lipschitzian properties of multifunctions. Nonlinear Anal. 25 (1995) 1401-1428.

[23] K.F. Ng and X.Y. Zheng, Error bounds for lower semicontinuous functions in normed spaces. SIAM J. Optim. 12 (2001) 1-17.

[24] S. Simons, Subdifferentials of convex functions. Contemp. Math. 204 (1997) 217-246.

[25] M. Studniarski and D.E. Ward, Weak sharp minima: characterizations and sufficient conditions. SIAM J. Control Optim. 38 (1999) 219-236.

[26] Z. Wu and J. Ye, On error bounds for lower semicontinuous functions. Math. Program. 92 (2002) 301-314.

[27] Z. Wu and J. Ye, First-order and second-order conditions for error bounds. Preprint (2002).

[28] C. Zălinescu, Weak sharp minima, well behaving functions and global error bounds for convex inequalities in Banach spaces, in Optimization Methods and their Applications, V. Bulatov and V. Baturin Eds., Irkutsk, Baikal (2001) 272-284.

[29] C. Zălinescu, Convex Analysis in General Vector Spaces. World Scientific Publ. Co., River Edge, NJ (2002). 